\title{
Application of Forensic DNA Identification to Human Cell Line Authentication
}

\section{Kaori Shintani-Ishida*}

Department of Forensic Medicine, Graduate School of Medicine, The University of Tokyo, 7-3-1 Hongo, Bunkyo-ku, Tokyo 113-0033, Japan

Short Tandem Repeat (STR) profiling has begun to be used in cell line authentication, in addition to its use in forensic casework. A database of STR profiles is a major practical tool for detection of cross-contamination or misidentification of cell lines, which is a serious problem that has largely been disregarded for over 50 years. In this issue of the Journal of Forensic Research, Fang et al. [1] describe STR genotyping using the AmpFlSTR ${ }^{\circledR}$ Identifiler $^{\circledR}$ kit in human and embryonic cell lines from the National Cancer Institute (NCI) and American Type Culture Collection (ATCC), and compare the resulting STR profiles with the ATCC database [1]. The extremely high concordance rate demonstrated that STR analysis is a useful tool for human cell line authentication. Amplification artifacts such as stutter products, allele drop out, and multiple allele profiles were observed, but the possibility of cross-contamination was excluded by performing two amplification reactions using two template DNAs.

Despite this good outcome, there are some remaining concerns about genetic alteration of STR loci during sub-culture. One cell line showed a STR profile that differed from that in the ATCC database. These results were probably caused by a stutter product, but the possibility of mutation could not be ruled out. Two pairs of cell lines each had the identical STR profiles except at one or two loci, suggesting that the cells originated from relatives or had STR profiles changed by mutations. Two tumors originating from the same individual showed different results at two loci, and one cell line showed a multiple allele profile. STR loci have a high mutation rate [2] and microsatellite instability is often observed in cancer cells [3]. In some cell lines, STR profiles can markedly alter during sub-culture [4]. Therefore, sub-lines of the same cell line may not have identical STR profiles.

In cell line authentication, when a STR profile is obtained that differs from the ATCC database at a few loci, there is a need to determine whether this is caused by genetic alteration or misidentification and to decide which method to use for this purpose. Few autosomal STR markers are available to add to the AmpFISTR ${ }^{\mathbb{R}}$ Identifiler ${ }^{\mathbb{R}}$ kit. However, some reports have shown that single nucleotide polymorphisms (SNPs) are useful supplementary markers in relationship-testing cases where genetic inconsistencies are detected between a child and the alleged parents [2,5]. Since SNP analysis constructed even with 52 loci has lower discrimination power than 15 STRs, an SNP-based strategy is unlikely to be a substitute for a STR-based method [6]. However, SNPs have a much lower overall mutation rate compared with STRs, suggesting that use of SNPs reduces the risk of an ambiguous genotype arising from mutation. Thus, the addition of SNP data resolved an analysis that had remained ambiguous even with extended STR typing [2]. Furthermore, the markedly different mutation rates of STRs and SNPs may help with interpretation of inconsistent STR results.

At present, the primary method for human cell line authentication is STR profiling. However, SNP analysis may help to resolve ambiguous STR results and it is likely that SNP markers will be validated for cell line identification, together with STRs. To achieve this, it will require overcoming many challenges, including standardization of a SNP analysis method for human identification, such as the SNPforID 52plex assay [7], and its development into a commercial kit; accumulation of know-how for interpretation of the resulting data; and verification of genetic stability during sub-culture. The wealth of experience available from forensic casework will be of great importance in establishing application of SNP profiling to cell line authentication, as a complement to STR profiling.

\section{References}

1. Fang R, Shewale JG, Nguyen VT, Cardoso H, Swerdel M, et al. (2011) STR Profiling of human cell lines: challenges and possible solutions to the growing problem. J Forensic Res S2-005.

2. Phillips C, Fondevila M, Garcia-Magarinos A, Rodriguez A, Salas A, et al (2008) Resolving relationship tests that show ambiguous STR results using autosomal SNPs as supplementary markers. Forensic Sci Int Genet 2: 198204

3. de la Chapelle A (2003) Microsatellite instability. N Engl J Med 349: 209-210.

4. Parson W, Kirchebner R, Mühlmann R, Renner K, Kofler A, et al. (2005) Cancer cell line identification by short tandem repeat profiling: power and limitations. FASEB J 19: 434-436.

5. Børsting C, Morling N (2011) Mutations and/or close relatives? Six case work examples where 49 autosomal SNPs were used as supplementary markers. Forensic Sci Int Genet 5: 236-241.

6. Amorim A, Pereira L (2005) Pros and cons in the use of SNPs in forensic kinship investigation: a comparative analysis with STRs. Forensic Sci Int 150: 17-21.

7. Musgrave-Brown E, Ballard D, Balogh K, Bender K, Berger B, et al. (2007) Forensic validation of the SNPforlD 52-plex assay. Forensic Sci Int Genet 1 186-190.

*Corresponding author: Kaori Shintani-Ishida, Department of Forensic Medicine Graduate School of Medicine, the University of Tokyo, 7-3-1 Hongo, Bunkyo-ku, Tokyo 113-0033, Japan, Tel: +81-3-5841-3367; E-mail: kaori@m.u-tokyo.ac.jp

Received April 11, 2012; Accepted April 11, 2012; Published April 13, 2012

Citation: Shintani-Ishida K (2012) Application of Forensic DNA Identification to Human Cell Line Authentication. J Forensic Res 3:e106. doi:10.4172/2157$7145.1000 \mathrm{e} 106$

Copyright: (c) 2012 Shintani-Ishida K. This is an open-access article distributed under the terms of the Creative Commons Attribution License, which permits unrestricted use, distribution, and reproduction in any medium, provided the original author and source are credited. 